\title{
Could Wallerian degeneration contribute to "leuko-araiosis" in subjects free of any vascular disorder?
}

D Leys, J P Pruvo, M Parent, P Vermersch, G Soetaert, M Steinling, A Delacourte, A Défossez, A Rapoport, J Clarisse, H Petit

\begin{abstract}
To determine the possible role of Wallerian degeneration secondary to the grey matter neuronal loss in the pathogenesis of "leuko-araiosis", computerised tomography (CT) of the brain was studied in 98 normotensive and non diabetic subjects free of cardiac diseases: 32 with Alzheimer's disease, 36 with Parkinson's disease, eight with progressive supranuclear palsy, and 22 controls. In Alzheimer's disease, leuko-araiosis scores were greater than in control subjects. Leuko-araiosis was more prominent in anterior periventricular areas in Parkinson's disease and progressive supranuclear palsy, and in posterior periventricular areas in Alzheimer's disease. In two patients with Alzheimer's disease and leuko-araiosis, necropsy revealed diffuse white matter pallor, mild fibrillary astrocytosis, and in one patient limited hyaline thickening of small white matter vessels, without any infarction or hypertensive change. Changes were more severe in white matter close to cortical areas with a great density of neurofibrillary tangles. Leuko-araiosis was more severe or more widespread in Alzheimer's disease than in Parkinson's disease, progressive supranuclear palsy and normal ageing. Differences in the location of leukoaraiosis between the four groups might be due to differences in the location of the grey matter disorder and Wallerian degeneration rather than amyloid angiopathy which has the same location in Alzheimer's disease, Parkinson's disease, progressive supranuclear palsy and normal ageing. Wallerian degeneration might be another cause of leuko-araiosis in neuro-degenerative disorders beside previously reported extra-cerebral predisposing factors and amyloid angiopathy.
\end{abstract}

Periventricular white matter lucencies, socalled leuko-araiosis, ${ }^{1}$ are sometimes reported in CT scans in healthy elderly subjects. ${ }^{23}$ They are correlated to age, mean systolic blood pressure, diabetes mellitus and previous cardiovascular diseases. ${ }^{25}$ They are also reported in demented patients, with a higher severity in multi-infarct dementia than in Alzheimer's disease. In demented patients they are correlated to the severity of the dementia and to the presence of other neurological abnormalities. ${ }^{6}$ In hypertensive subjects leuko-araiosis is usually attributed to subcortical vascular changes and the terms "subcortical arteriosclerotic encephalopathy" 59 or "Binswanger's disease" 10 are commonly applied.

Nevertheless, Rezek et $a l^{2}$ in five patients with leuko-araiosis and definite Alzheimer's disease, only found diffuse white matter pallor, mild fibrillary astrocytosis and hyaline mural thickening of small cerebral arteries, without any hypertensive vascular change nor infarction. In Alzheimer's disease the role of the cortical amyloid angiopathy in the pathogenesis of leuko-araiosis is frequently invoked $^{1}$ but the possible role of Wallerian degeneration secondary to the cortical neuronal loss has not been studied.

We undertook this study to determine the possible role of Wallerian degeneration in the pathogenesis of leuko-araiosis using a carefully selected population of subjects free from any previously reported predisposing factors of leuko-araiosis.

Subjects and methods

Subjects

Ninety eight subjects were included in this study: 32 with Alzheimer's disease, 36 with Parkinson's disease, eight with progressive supranuclear palsy, and 22 controls. To avoid subjects with risk factors shown to be associated with leuko-araiosis ${ }^{235911}$ and to compare homogenous groups differing only by their neurological state (Alzheimer's disease, Parkinson's disease, progressive supranuclear palsy, controls), we only included normotensive and non diabetic subjects free of cardiac diseases. Hypertension was defined using the criteria by Inzitari et al. ${ }^{4}$ Diabetes mellitus was defined as the presence of one or more of the following: 1) a glucose blood level greater than $1.05 \mathrm{~g} / 1$ using the glucose oxidase technique, 2) a previous medical diagnosis of diabetes mellitus and 3 ) previous or current treatment using antidiabetic drugs. Cardiac disorders were defined as the presence of any abnormality on clinical examination or electrocardiogram, except a right bundle branch block. None of these 98 subjects had evidence of a neuro-degenerative disorder other than Alzheimer's disease, Parkinson's disease or progressive supranuclear palsy; they were free of toxic disorders, focal cerebrovascular disease, or any metabolic disorder involving the glia. None of them had abnormal motor find- 
ings, symptomatic infarctions or small focal lucencies consistent with lacunar infarcts, according to Steingart's criteria. ${ }^{36}$

The diagnosis of probable Alzheimer's disease was made according to the NINCDSADRDA work group criteria. ${ }^{12}$ The Alzheimer's disease group included 20 women and 12 men with a mean age 65.63 (range, 51 to 80 ), with mean Mini Mental State ${ }^{13}$ (MMS) scores of 11.34 (range, 0 to 23). None of them had myoclonus or extra-pyramidal disorder. In 24 out of the 32 patients, Alzheimer's disease began before the age of 65 . None of them had a definite familial history of Alzheimer's disease, but 10 patients had one parent affected by dementia, without any other information. They scored two or less on the modified Hachinski's vascular score. ${ }^{14}$ Several patients have been included in previous studies of MRI white matter changes in Alzheimer's disease. ${ }^{15}$

The diagnosis of Parkinson's disease was established according to the Adams' and Victor's standard criteria. ${ }^{16}$ This group included 23 women and 13 men, mean age $68 \cdot 17$ (range, 50 to 81 ) with mean MMS scores of 25.58 (range, 15 to 30 ).

The diagnosis of progressive supranuclear palsy was established according to Blin's criteria of definite progressive supranuclear palsy. ${ }^{17}$ This group included five women and three men, mean age 71.75 (range, 66 to 83 ) with mean MMS scores of 20.63 (range, 15 to 27).

Control subjects were patients aged 50 or more, examined over a period of five months. They had a disease which is not known to modify morphological brain structures: migraine (eight cases), idiopathic trigeminal neuralgia (two cases), temporal arteritis (two cases), Ménière's syndrome (three cases), spinal neurinoma (one case), long term sequelae of a bacterial dorsal epiduritis (one case), spinal compression by cervical arthrosis (one case), and Bell's palsy (four cases). We excluded patients with the clinical diagnosis of multiple sclerosis, neoplasm, stroke, trauma, hydrocephalus and neurodegenerative disorders. This group included 15 women and seven men, mean age 65.96 (range, 50 to 87 ), with MMS scores greater than 27 (means 29.09; range, 28 to 30 ).

Statistical analysis failed to reveal any difference between the four groups of subjects for sex (Chi square test) and age (ANOVA 1 way).

\section{Methods}

CT scans

Non-contrast CT scans were carried out on CE 12000 (CGR, France) or on Siemens Somatom II (Siemens, West Germany) using $10 \mathrm{~mm}$ contiguous slice thickness in a plane $15^{\circ}$ negative to the cantho-meathal plane. Scan times were $9 \cdot 6 \mathrm{~s}$ per slice. The scans were interpreted from films using Rezek's method ${ }^{2}$ for systematic evaluation of leuko-araiosis in 15 areas in each hemisphere, including 14 white matter regions and the basal ganglia-thalamic regions, of four CT slices. According to
Rezek's study ${ }^{2}$ the areas that were examined were located as follows: 1) at the level of the basal ganglia: a (superficial frontal), b (deep frontal), c (basal ganglia), d (parietal), e (occipital); 2) at the level of the body of the lateral ventricles: $f$ (superficial frontal), $g$ (deep frontal), $h$ (deep parietal), i (superficial parietal); 3) at the level of the top of the lateral ventricles: $j$ (frontal anterior), $k$ (frontal posterior), 1 (parietal anterior), $\mathrm{m}$ (parietal posterior); 4) at the level of the vertex: $n$ (anterior), o (posterior).

An area was considered lucent if the density was between that of normal white matter and cerebrospinal fluid (CSF). Severity of a lucency was scored from 0 (no lucency) to 6 (severe lucency wider than $2 \mathrm{~cm}^{2}$ ), according to Rezek's scoring system ${ }^{2}$ based on size $\left(<2 \mathrm{~cm}^{2},=2 \mathrm{~cm}^{2}\right.$ or $\left.>2 \mathrm{~cm}^{2}\right)$ and degree (absent, mild or severe) of contrast difference with surrounding brain. Lucencies due to artefacts were excluded. Ratings were separately performed by two observers (DL and JPP) without knowledge of diagnosis, ages and MMS scores. The "leuko-araiosis" score ("LA" score) was the sum of scores given by each observer, and was ranged from 0 to 360 (2 observers/15 areas/2 hemispheres/maximum score of 6 by area). The postero-anterior assymetry was assessed by the ratio of the difference of posterior minus anterior "leukoaraiosis scores" divided by "leuko-araiosis score" (LAp-LAa)/LA); LAp is the sum of LA scores in posterior areas of both hemispheres, corresponding to the areas d-e-h-i-l-m-o as defined by Rezek $e t a l^{2}$ and LAa is the sum of LA scores in anterior areas, corresponding to the areas $a-b-f-g-j-k-n$ of both hemispheres.

\section{Neuropathological correlates}

We studied the white matter in seven regions using hematoxylin-eosin, Luxol and Bodian staining methods, and immunohistochemistry with anti-GFAP antibodies (Laboratory Dako); white matter lesions were ranked as mild, moderate or severe, according to Brun's and Englund's criteria. ${ }^{18}$ In the related cortex, senile plaques detected by Bodian and neurofibrillary tangles detected by antineurofibrillary tangles antibodies were regionally counted using Iseki's method. ${ }^{19}$

\section{Statistical analysis}

Kruskall-Wallis analysis of variance was performed to compare 1) leuko-araiosis scores, 2) (LAp-LAa)/LA scores, and 3) regional scores of leuko-araiosis in the 30 studied areas (sum of the scores given by the two raters in each studied area) of the four groups. When a significant difference was found within the four groups, we used the non parametric $U$ test of Mann and Whitney to compare groups 2 by 2 . Spearman Rank correlation test was performed to study correlations between leuko-araiosis scores, the ages in the control group and the MMS scores in the Alzheimer's disease group. The level of interobserver agreement for CT analysis was measured on a sample of $40 \mathrm{CTs}$ analysed by four observers (DL, JPP, PV, AR) by Kappa, separately for each single area, using 
the Fleiss method. ${ }^{20}{ }^{21}$ It has been suggested ${ }^{22}$ that agreement can be considered excellent when Kappa $>0.80$. The level of intraobserver agreement was measured on another sample of $30 \mathrm{CTs}$ analysed again by the two observers (DL and JPP) without knowledge of their first scores, using the same Fleiss method. ${ }^{20}$ Kruskall-Wallis analysis of variance was performed to compare, in the brains of patients with Alzheimer's disease, the number of senile plaques and neurofibrillary tangles between three severity groups by white matter involvement. ${ }^{18}$

Modalities of collection, computerisation and storage of data were approved by the Regional Ethical Committee both for patients and control subjects.

\section{Results}

CT leuko-araiosis analysis

The study of inter- and intra-observer agreements provided the following values: the levels of inter- and intra-observer agreement determined by Kappa were respectively 0.881 for the former, 0.942 (DL) and 0.949 (JPP) for the last.

Leuko-araiosis scores were significantly different between the four groups $(p<0.02)$, with greater leuko-araiosis scores in $\mathrm{Alz}$ heimer's disease than in Parkinson's disease $(\mathrm{p}<0.02)$ and controls $(\mathrm{p}<0.05)$ without any significant difference between other groups (fig 1). It has been possible to establish the values of the index of assymmetry in the 43 subjects (18 Alzheimer's disease, 10 Parkinson's disease, seven progressive supranuclear palsy, eight controls) with leuko-araiosis scores different from 0 . The index (LAp-LAa)/LA was significantly different between the four groups ( $p<0.01$ ) with lower scores (that is more anterior leuko-araiosis) in progressive supranuclear palsy than in Alzheimer's disease $(\mathrm{p}<0.001)$, Parkinson's disease $(\mathrm{p}<0.05)$ and controls $(p<0.01)$ and in Parkinson's disease than in Alzheimer's disease $(p<0.05)$, without any significant difference between the other groups (fig 2). The regional scores of leuko-araiosis were significantly different between the four groups in areas "a"

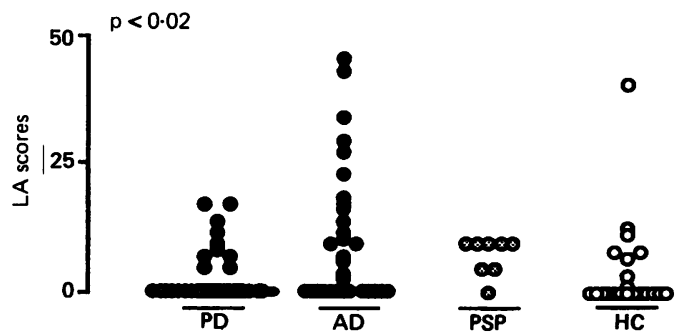

Figure 1 Values of leuko-araiosis scores in subjects with Alzheimer's disease (AD), Parkinson's disease (PD), progressive supranuclear palsy (PSP) and healthy elderly controls (C). Each point represents the "leukoaraioisis" (LA) score of one subject. Leuko-araiosis scores were significantly different $(p<0.02)$ between the four groups (Kruskall-Wallis $H$ test), with greater values in $A D$ than in $P D(p<0.02)$, and controls $(p<0.05)$, and without any difference between the $p<0.05)$, and without any difference betwe
other groups (Mann and Whitney $U$ test).

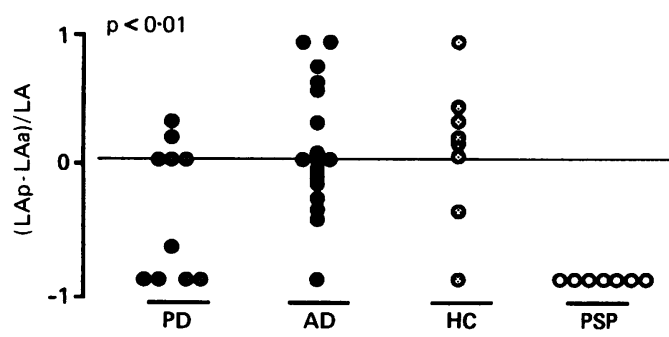

Figure 2 The index of posterior/anterior asymmetry, $(L A p-L A a) / L A$, was significantly different $(p<0.01)$ between the four groups (Kruskall-Wallis $H$ test) with lower values in progressive supranuclear palsy than in Alzheimer's disease ( $p<0.001)$, Parkinson's disease $(p<0.05)$ and controls $(p<0.01)$ and in Parkinson's disease than in Alzheimer's disease $(p<0.05)$, without any significant difference between the other groups (Mann and Whitney $U$ test).

$(P<0.001)$, "b" $(P<0.01)$ and " $h$ " $(P<0.05)$. In the anterior areas " $a$ " and " $b$ ", the regional scores of leuko-araiosis were greater in progressive supranuclear palsy than in each other group ( $p<0.001)$; in the posterior area "h", the regional score of leukoaraiosis was greater in Alzheimer's disease than in Parkinson's disease $(\mathrm{p}<0.01)$, progressive supranuclear palsy ( $\mathrm{p}<0.05)$ and normal controls $(\mathrm{p}<0.05)$. For areas without differences between the four groups the $B$ risk of type II error was higher than $0 \cdot 20$.

Leuko-araiosis scores were correlated to age in controls $\left(r_{s}=0.604 ; p<0.01\right)$, but were not correlated to MMS scores in Alzheimer's disease $\left(r_{\mathrm{s}}=0 \cdot 25 ; \mathrm{p} \# 0 \cdot 15\right)$.

\section{Neuropathological analysis}

Pathological examination was performed in three patients with Alzheimer's disease, who died at the age of 57,75 and 76 with leukoaraiosis scores respectively 8,4 and 14 . In these three cases Alzheimer's disease was pathologically confirmed. The degree of Alzheimer's disease changes in these three necropsy subjects was sufficient to explain the dementia. White matter could not be studied in the younger patient who died from two voluminous cerebral haemorrhages secondary to amyloid angiopathy lesions one year after he had been included in this study.

The white matter of the two remaining patients was characterised in both cases by diffuse myelin pallor and mild fibrillary astrocytosis. One patient had hyaline thickening of small vessels. Both patients had signs of amyloid angiopathy strictly limited to the cortical and leptomeningeal vessels, but the white matter vessels were left free of any lesion of amyloid angiopathy. The two patients had no evidence of brain infarct or hypertensive vascular change.

In these two patients, the severity of white matter changes did not differ whatever the number of senile plaques, but was greater in white matter areas adjacent to cortical areas with numerous neurofibrillary tangles $(\mathrm{p}<0.05)$ (fig 3).

\section{Discussion}

Our study revealed that 1) in Alzheimer's 
Figure 3 Study of the severity of white matter changes, using Brun's criteria, ${ }^{18}$ as a function of the number of

neurofibrillary tangles and

of the number of senile

plaques (senile plaques), using Iseki's criteria, ${ }^{19}$ in

the adjacent cortex. The severity of white matter changes did not differ whatever the number of senile plaques, but was greater in white matter areas adjacent to cortical areas with numerous neurofibrillary tangles $(p<0.05)$.

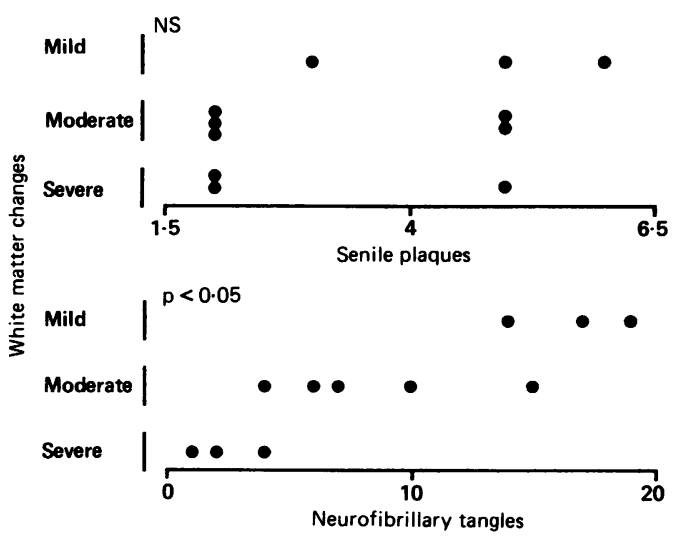

disease leuko-araiosis scores were greater than in control subjects, 2) leuko-araiosis was more prominent in anterior periventricular areas in Parkinson's disease and progressive supranuclear palsy, and in posterior periventricular areas in Alzheimer's disease, 3) in two patients with Alzheimer's disease and leuko-araiosis necropsy revealed diffuse white matter pallor, mild fibrillary astrocytosis, and in one case, limited hyaline thickening of small white matter vessels, without any infarction or hypertensive change, 4 ) changes were more severe in white matter close to cortical areas with a great density of neurofibrillary tangles. Previous CT studies, with the exception of Rezek's, ${ }^{2}$ did not attempt to quantify the severity of leukoaraiosis: most authors separated subjects with leuko-araiosis from subjects without. To our knowledge such a study in Parkinson's disease and progressive supranuclear palsy has not been previously performed and no other studies have attempted to account for the topography of leuko-araiosis.

To our first question (that is, is Alzheimer's disease a possible factor of leuko-araiosis once confusing factors are eliminated?) our study suggests a positive answer, because we found greater leuko-araiosis scores in Alzheimer patients than in control subjects. According to previous studies ours also suggested that the neuropathological lesions of Alzheimer's disease might be a predisposing factor of leukoaraiosis. ${ }^{27}$ Since one of our necropsy patients had hyaline thickening of the white matter vessels we cannot exclude, in spite of severe criteria of exclusion, that this patient had a slight hypertension which could only have been detected using a continous recording of blood pressure. However, we think that such a bias is very unlikely though we cannot exclude it.

To our second question (that is, has Wallerian degeneration a possible role in the pathogenesis of leuko-araiosis in Alzheimer's disease?) our study also suggests a positive answer. At necropsy our patients had signs of amyloid angiopathy limited to cortical vessels but sparing the white matter, as previously reported in five cases by Rezek. ${ }^{2}$ The cortical blood supply is richly served by collaterals so that if a cortical vessel suffering amyloid deposits is occluded, adjacent grey matter may be supplied by adequate collateral flow. ${ }^{2}$ The white matter served by the same artery may suffer ischaemic damage since once the artery penetrates the white matter it has no collaterals. ${ }^{18}$ Responsibility of the amyloid angiopathy cannot be excluded by our study: Alzheimer's disease patients might have had more severe leuko-araiosis than controls because amyloid angiopathy is usually more frequent and more severe in Alzheimer's disease. If amyloid angiopathy was the main factor of leuko-araiosis in Alzheimer's disease, the mechanism of "incomplete infarction" previously suggested by Brun and Englund ${ }^{18} 23$ and more recently by Hachinski et al ${ }^{124}$ would not explain why leuko-araiosis is more prominent in anterior areas in Parkinson's disease and progressive supranuclear palsy, since cortical amyloid angiopathy has, to our knowledge, the same location in Alzheimer's disease and in normal ageing and usually only differs in its severity.

Another factor therefore is necessary to explain differences in the location of leukoaraiosis between Alzheimer's disease, on one hand, and progressive supranuclear palsy or Parkinson's disease, on the other. In Parkinson's disease we found a slight predominance of leuko-araiosis in anterior areas, sometimes clinically affected in Parkinson's disease, though available information is contradictory on the subject. ${ }^{25}$ In progressive supranuclear palsy we found a strong predominance of leuko-araiosis in anterior areas, which usually are severely affected, as suggested by clinical $^{26}$ and metabolic ${ }^{27}{ }^{28}$ studies. Differences in the location of leuko-araiosis between the four groups might be explained by a decrease of myelin density secondary to the Wallerian degeneration due to the grey matter disorder, which is mainly cortical, more widespread and more posterior in Alzheimer's disease, and mainly subcortical and more anterior in progressive supranuclear palsy and perhaps in Parkinson's disease. In Alzheimer's disease the pathological changes in the cortex are more severe in parieto-temporal cortex and hippocampus which send fibres to the posterior association areas. In our Alzheimer's patients the area " $h$ " as defined by Rezek et $a l^{2}$ was the most affected by leuko-araiosis. This is one of the white matter areas closest to the most affected cortical area in Alzheimer's disease. This is compatible with the hypothesis that white matter changes in Alzheimer's disease could be secondary to Wallerian degeneration. Nevertheless these data suggest that: 1) CT scan provides a resolution too low to study small white matter areas that are closest to the hippocampus, 2) in other areas no differences have been found between the groups but the small number of subjects might explain a high level of type II error and we cannot exclude a difference.

If leuko-araiosis is partially secondary to a neuronal drop-out we would expect the eight control subjects with leuko-araiosis to have cognitive impairment; their neuropsychological examinations, however, were limited to the Folstein's $M_{M M S^{13}}$ which might have insufficient sensitivity to detect "preclinical" Alzheimer's disease. Moreover, we found a ten- 
dency to a negative correlation between leukoaraiosis scores and MMS in Alzheimer's disease, but it did not reach a statistical level of significance. If leuko-araiosis is secondary to a neuronal drop-out we would expect that, in the eight control subjects with leuko-araiosis, this change would herald the subsequent development of dementia. Only further longitudinal studies could answer this important question.

We thank T McBride for his helpful comments.

This study was supported by grants from the INSERM (grant CAR 489016), the University Lille II, and the "Sociète de medecine du Nord".

1 Hachinski VC, Potter P, Merskey H. Leuko-Araiosis. Arch Neurol 1987;44:21-3.

2 Rezek DL, Morris JC, Fulling KH, et al. Periventricular white matter lucencies in senile dementia of the Alzheimer type and in normal ageing. Neurology 1987;37:1365-8.

3 Steingart A, Hachinski VC, Lau C, et al. Cognitive and neurologic findings in subjects with diffuse white matter lucencies on computed tomographic scan (leuko-araiosis). Arch Neurol 1987;44:32-5.

4 Inzitari D, Diaz F, Fox A, et al. Vascular risk factors and leuco-araiosis. Arch Neurol 1987;44:42-7.

5 Kinkel WR, Jacobs L, Polachini I, et al. Subcortical arteriosclerotic encephalopathy (Binswanger's disease): computed tomographic, nuclear magnetic resonance, computed tomographic, nuclear magnetic reson

6 Steingart A, Hachinski VC, Lau C, et al. Cognitive and neurological findings in demented patients with diffuse white matter lucencies on computed tomographic scan (leuko-araiosis). Arch Neurol 1987;44:36-9.

7 Gupta SR, Naheedy MH, Young JC, et al. Periventricular white matter changes and dementia. Clinical, neuropsychological, radiological and pathological correlations. Arch Neurol 1988;45:637-41.

8 Aharon-Peretz J, Cummings JL, Hill MA. Vascular dementia and dementia of the Alzheimer type. Cognition, ventricular size and leuco-araiosis. Arch Neurol 1988; 45:719-21.

9 Loizou LA, Kendall BE, Marshall J. Subcortical arteriosclerotic encephalopathy: a clinical and radiological invessclerotic encephalopathy: a clinical and radiological inves-

10 De Reuck J, Crevits L, De Coster W, et al. Pathogenesis of Binswanger's chronic subcortical encephalopathy. Neurology 1980:30:920-8.

11 Huang K, Wu L, Luo Y. Binswanger's disease: progressive subcortical encephalopathy or multi-infarct dementia? Can J Neurol Sci 1985;12:88-94.

12 McKahnn G, Drachman D, Folstein M, et al. Clinical diagnosis of Alzheimer's disease: Report of the NINCDS ADRDA Work group under the auspices of department of health and human services task force on Alzheimer's disease. Neurology 1984;34:939-44.

13 Folstein MF, Folstein SE, McHugh PR. "Mini-Mental state". A practical method for grading the cognitive state
of patients for the clinician. J Psychiat Res 1975;12: of patients

14 Loeb C, Gandolfo C. Diagnostic evaluation of degenerative and vascular dementia. Stroke 1983;14:399-401.

15 Leys D, Soetaert G, Petit $H$, et al. Periventricular and white matter magnetic resonance hyperintensities do not differ between Alzheimer's disease and normal aging. Arch Neurol 1990;47:521-7.

16 Adams RD, Victor M. Principles of neurology, 3rd ed. New York: McGraw-Hill: 1985:874-9.

17 Blin J, Baron JC, Dubois B, et al. Depression of regional brain energy metabolism in progressive supranuclear palsy and clinico-metabolic correlations. J Cereb Blood Flow Metab 1989;9:S21.

18 Brun A, Englund E. A white matter disorder in dementia of the Alzheimer type: a pathoanatomical study. Ann Neurol the Alzheimer type:

19 Iseki E, Matsushita M, Kosaka K, et al. Distribution and morphology of brain stem plaques in Alzheimer's disease. Acta Neuropathol 1989;78:131-6.

20 Fleiss JL, Nee JCM, Landis JR. Large sample variance of Kappa in the case of different sets of raters. Psychol Bull 1979;86:974-7.

21 Fleiss JL. Measuring nominal scale agreement among many raters. Psychol Bull 1971;76:378-82.

22 Theodossi A, Skene AM, Portmann B, et al. Observer variation assessment in liver biopsies including analysis by Kappa statistics. Gastroenterology 1980;79:232-41.

23 Englund E, Brun A, Alling C. White matter changes in dementia of Alzheimer's type: biochemical and neuropathological correlates. Brain 1988;111:1425-39.

24 Janota I, Mirsen TR, Hachinski VC, et al. Neuropathologic correlates of Leuko-Araiosis. Arch Neurol 1989;46: 1124-8.

25 Taylor AE, Saint-Cry JA, Lang AE. Frontal lobe dysfunction in Parkinson's disease. The cortical focus of neostriatal outflow. Brain 1986;109:845-83.

26 Cambier J, Masson M, Viader F, et al. Le syndrome frontal de la paralysie supranucléaire progressive. Rev Neurol (Paris) 1985;141:528-36.

27 D'Antona R, Baron JC, Samson Y, et al. Subcortical dementia: frontal cortex hypometabolism detected by positron tomography in patients with progressive supranuclear palsy. Brain 1985;108:785-99.

28 Goffinet AM, De Volder AG, Gillain C, et al Positron tomography demonstrates frontal lobe hypometabolism in progressive supranuclear palsy. Ann Neurol 1989;25: progressi $131-9$.

29 Delacourte A, Lenders MB, Défossez A, et al. Neuropathologie et neurobiologie de la maladie d'Alzheimer. In: Leys D, Petit H eds. La maladie d'Alzheimer et ses limites. Paris: Masson, 1988:103-13. 